\title{
Stroke Prediction Context-Aware Health Care System
}

\author{
Hamid Mcheick \\ Department of Computer Science \\ University of Quebec at Chicoutimi \\ UQAC \\ Chicoutimi, Canada \\ Hamid_Mcheick@uqac.ca
}

\author{
Hoda Nasser, Mohamed Dbouk \\ Faculty of Sciences (I) \\ Lebanese University \\ Beirut, Lebanon \\ Hoda_nasser@homail.com; \\ mdbouk@ul.edu.lb
}

\author{
Ahmad Nasser \\ Specialist in family medicine \\ American University of Beirut \\ Beirut, Lebanon, \\ alnasser@gmail.com
}

\begin{abstract}
This paper proposes a prediction framework based on ontology and Bayesian Belief Networks BBN to support a medical teams in every daily. We propose a Stroke Prediction System (SPS), a new software component to handle the uncertainty of having a stroke disease by determining the risk score level. This is composed of four layers: acquisition of data, aggregation, reasoning and application. SPS senses, collects, and analyzes data of a patient, then uses wearable sensors and the mobile application to interact with the patient and staffs. When the risk reaches critical limits, SPS notifies all concerned parties; the patient, the doctor, and the emergency department. The patient profile is also updated to reflect this urgent intervention requirement. A Bayesian model is designed and implemented using the Netica tool to prove its efficiency i) by handling patient context remotely and verifying its changes locally and ii) on predicting missing probabilities and calculate the probability of high risk level for emergency cases. The SPS system improves the accuracy of decision making and uses a new ontology of stroke disease inspired from our Parkinson ontology already developed.
\end{abstract}

Keywords-Healthcare; context awareness, Ubiquitous computing; Context-aware; ontology; probabilistic model; sensors

\section{INTRODUCTION}

Ubiquitous computing connects a user to his environment, being present everywhere and in real-time using many kind of devices such as smart phones and sensors. It is an emergent technology of both knowledge and information dissemination. Its main purpose is to adapt intelligently to the user's context. The user can query this intelligent system which instantly responds according to several parameters (context). This system could help medical teams to solve many challenges in systemizing patient's data, and making a decision when there is a need to. While attempting to make a diagnostic decision; Nurses and doctors need to deal with huge amount of information extracted from the patient's medical record and handle uncertainty in the meantime. One of the solution is the context aware systems which provide a clear improvement in medical monitoring and decision making systems [3].
However, medical context-aware systems can't always identify the current context precisely, and they may face non-absolute rule. This uncertainty must be handled, especially when patient is doing an activity remotely. Uncertainty is a situation of inadequate information [12] which can be of three sorts: inexactness, unreliability, and border with ignorance. Many context modeling and retrieval architectures tend to over-simplify uncertainty by assuming a perfect knowledge in combination with perfect inference [1].

One of the most representation model of context is the ontology, which has properties of rich expressiveness to describe the relationships between the vocabularies of the context knowledge. Both ontology and Bayesian network are used to deal with inference and to handle uncertainty. In our study ontology represents learn directed causal relationships [1]. We designed and implemented new Stroke Predicting System (SPS) to predict the risk of a stroke that may affect patient Transient Ischemic Attack (TIA), determine its level of stroke risk, and notify the patient and his medical team. This team deals with status based on SPS' prediction remotely using our server (similar to cloud) and verifies its changes locally before sending messages through the network. This paper is organized as follows: section 2 discusses some related work, section 3 describes our motivation and problem in details. Section 4 describes the Stroke Prediction System architecture and Model. Section 5 illustrates the implementation, results and interpretation of the study based on Netica software and real data. Finally section 6 summarizes what we have done and gives directions for future works.

\section{RELATED WORK}

All around the world, the population and the number of elderly people are increasing, which putts an enormous pressure on health systems. The relevant patient and environmental data can be collected and interpreted in context aware system that improves the quality of medical care using patient monitoring context aware application.

Decision making in medicine diagnosing is a hard and challenging task because of countless symptoms and diseases, incomplete knowledge and imperfection medicine 
rules. This fast real-time decision can be offered by the clinical decision support system (CDSS).

CDSS is built based on Bayesian network [3] but it can be built using neural network or genetic algorithms, too. It would be a part of system designed for cardiac telerehabilitation for patients after infarction. The system replaces a rule-based variant that had been used to control the patient's exercise session, including the generation of alarms [3]. The evaluation shows that the number of false alarm can be reduced by introducing of Bayesian networks.

Teles et al. use also the Bayesian networks to improve the decision-making process in public health systems [4]. The author presents LARIISA_Bay, a context-aware platform to support applications in public health systems. The main purpose of this system is to assist teams of health specialists so they can better diagnose diseases through data collected from patients using LARIISA [4].

CDSS and LARIISA_Bay are monitoring and decision making systems, they monitor, detect and make the good decision at the right time, but cannot predict the medical issue before its occurrence. Our stroke prediction system (SPS) is designed to use the monitoring results of a patient in the prediction of a stroke attack; it aims to propose the necessary treatment to save the patient from any stroke attack.

Bayes' Theorem is a simple mathematical formula used for calculating conditional probabilities. The key feature of BBNs is that they enable us to model and reason about uncertainty. The BBN forces the assessor to expose all assumptions about the impact of different forms of evidence and hence provides a visible and auditable dependability or safety argument [11]. Therefore we can say that Bayesian model can be pretty much everywhere.

\section{DEFINITION AND MOTIVATION}

A stroke occurs when blood flow to a part of the brain stops. A stroke is sometimes called "brain attack". There are two major types of stroke, Ischemic stroke and Hemorrhagic stroke [2]. One of causes of ischemic stroke is the disturbance of blood flow through carotid arteries.

Transient Ischemic Attack (TIA) is known as ministroke. The only difference between a stroke and TIA is that with TIA the blockage is transient and temporary, while with stroke the blockage is permanent. A TIA can decrease your life expectancy by up to 20 percent and immediate medical attention is necessary to help prevent your risk of stroke. So TIA is a warning sign for future stroke.

Several Risk Scores have been developed to predict the stroke risk after Transient Ischemic Attack (TIA). The original ABCD score is one of the most famous applicable risk scores [7]. In 2007, Purroy et al. [5] suggested a new risk score called ABCD2, then in 2010, Lavallée et al. [6] suggested two new risk scores to predict stroke occurrence earlier in time after a patient suffers from TIA. The two new risk scores are named ABCD3 and ABCD3-1. As shown in [7] $A B C D 3-I(c / i)$ is the best to use in order to predict the occurrence of a stroke using risk factors for TIA Patient during seven days after TIA.

As in [7] the following elements are the risk factors of stroke and each risk factor had a probability:

TABLE I. NODES OR PARAMETERS PROBABILITIES

$\left.\begin{array}{|l|c|c|}\hline \multicolumn{1}{|c|}{\text { Parameter }} & \text { Probability } & \text { coefficient } \\ \hline \text { Parameter } & \text { Probability } & \text { coefficient } \\ \hline \text { Age>60 } & 62.2 \% & 1 \\ \hline \text { Diabetes } & 23.5 \% & 2 \\ \hline \text { Dual TIA } & 19 \% & 2 \\ \hline \text { Stenosis } & 38.1 \% & 2 \\ \hline \text { Unilatery Weakness } & 59.3 \% & 2 \\ \hline \begin{array}{l}\text { Symptoms } \\ \text { Duration>10min }\end{array} & 35.8 \% & 2 \\ \hline \begin{array}{l}\text { Symptoms } \\ \text { Duration>60min }\end{array} & 43.6 \% & 1 \\ \hline \text { Speech Impairment } \\ \text { without Weakness }\end{array}\right)$

IV. SPECIFIC PROBLEM AND GOAL

In Emergency room and remote patient home, large number of people is diagnosed with TIA, so they must follow the necessary treatment. Studies had shown that urgent assessment and clinic treatment following TIA can result in an $80 \%$ reduction in subsequent stroke [8]. The study in [8] included 5106 patients, during a median length of stay of 5 days (interquartile range, 4-8), stroke recurrence (or stroke after TIA) occurred in 40 patients (0.8\%) and was independently associated with history of TIA [8].

On the other hand [9] and [10] show that during seven days, thirty days and ninety days after TIA the risks of stroke following TIA are estimated at 5.2\%, 8\% and 9.2\% respectively.

In order to calculate the risk score of a stroke following TIA, the risk score factors must be detected. Therefore, patients with TIA need to be observed. The observation of TIA patient in hospital may be difficult because it demands a huge number of beds for a long time due to the number of people that may have TIA which is a real problem.

Since all the methods and applications that try to detect Stroke Risk factors and predict the risk of stroke recurrence are human based, we propose a Stroke Prediction System (SPS), it is an automatic computerized context-aware health care system that monitors blood pressure, Carotid stenosis, 
and notice TIA symptoms of the patient with TIA history. Our system use the monitored data to calculate the risk score of having a stroke during the next seven days. In case the patient is at high risk, the system notifies him to go to the emergency, send a warning message to his doctor and update his profile on the server. Therefore our contribution aims to reduce stroke attacks, by trying to predict its occurrence, using Stroke Prediction System (SPS).

\section{SPS ARCHITECTURE AND MODEL}

\section{A. System Architecture and Ontology}

Stroke Prediction System (SPS) is a context aware system that tries to predict the stroke risk level based on patient context using ABCD3-1(c/i) model. Our proposed system is represented by four layers, physical (sensor) layer, aggregation, reasoning and application layers.

A sensor in the form of hand watch is used to measure the blood pressure of the patient, a two Doppler probes (used in carotid duplex ultrasound) are attached to the internal carotid artery and cerebral major artery in order to measure the velocity of blood flow into these arteries. The changes of context values are verified locally before sending any information through network.

In aggregation Layer, the patient is registered to the application by adding its profile with the necessary personal and medical information to be stored (age, diabetes, TIA history), while other data are received from patient sensors.

In reasoning layer we developed a mobile application responsible for making decision of a stroke risk in collaboration with our server. This application receives data from sensors every period of time, requests symptoms input from user if necessary and calculates stroke risk score by applying ABCD3-1(c/i) formula to the factors received from aggregation layer. Our server handles the reasoning issues. Then the application decides if the risk is high, medium or low based on a specific threshold. The application layer is represented by users, doctors and nurses. The period is depending of changes of context' values and intervals of symptoms.

In case the risk score of a stroke is high, patient must directly go to the emergency room to take the necessary treatment, or if risk score is medium the patient is notified to take a doctor appointment. These data of risk factors and calculated scores will be stored in the profile independent of the score results.

The profile of the user in his domain is modelled with the SPS ontology. It represents characteristics of a user profile that act as factors of the Risk score of a recurrent stroke. Those factors are considered as input parameters to SPS system that outputs the Risk level of a stroke. The classes used in our ontology are: patient, patient profile, pressure watch sensor, wearable carotid and cerebral stenosis detector, stroke factors, risk score, SPS Mobile application, doctor, server, and emergency room.
But ontology structure has no rich representation to analyze the risks and to represent our system; we extended it to a probabilistic model so it can handle uncertainty.

The task of building the structure and assigning the probability distributions of a Bayesian Network is complex and knowledge-intensive. Our work is influenced by our colleagues paper PHEN: Parkinson Helper Emergency Notification System using Bayesian Belief Network [1]. Our SPS models the entities of Stroke prediction system.

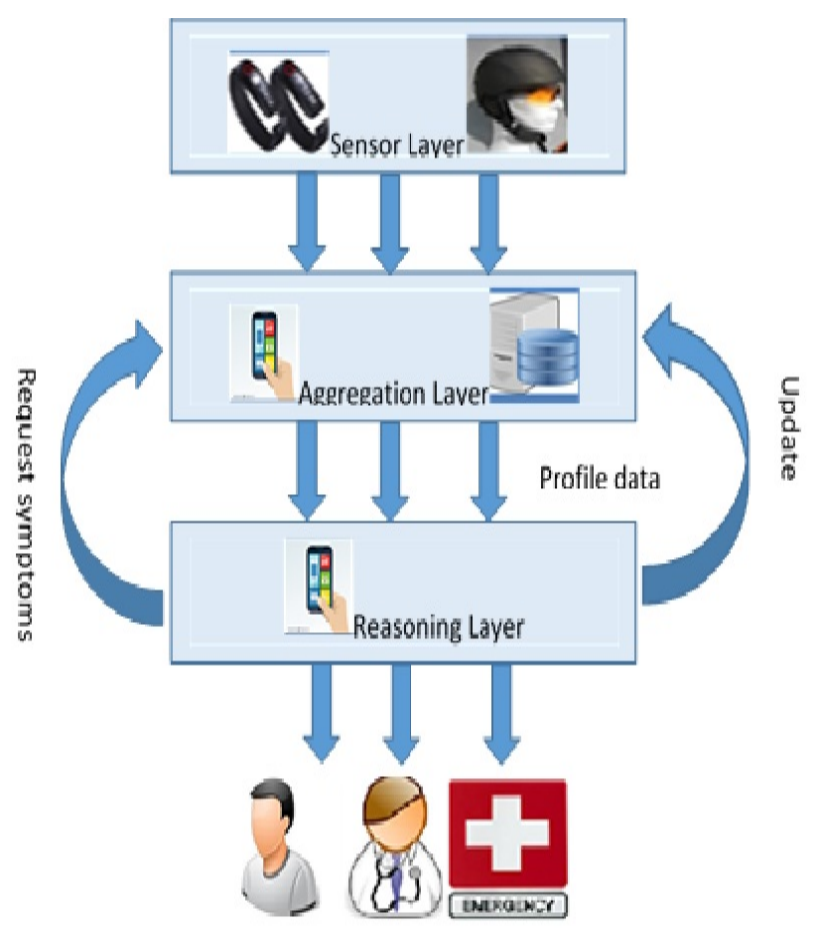

Figure 1. SPS architecture

\section{B. SPS Bayesian Belief Network Model}

Same as the PHEN paper [1], we generate our context ontology. Then a Bayesian network, Based on the probabilistic model and our specific ontology, is derived with structure of Bayesian belief network. The sensors detect the data which is sent to aggregation layer to verify them, then, sending them to reasoning layer.

\section{SPS IMPLEMENTATION}

Netica is a powerful tool that allows user to work easily with a Bayesian network model. We applied our Bayesian belief network (BBN) structure to Netica [14] by drawing classes as nodes, and identify relationships between them by drawing lines directed from parent node to the child node. Therefore we can use Netica also to find optimal values for parents' nodes which lead to the required value of a child node. We choose to apply the probabilities of risk factors for ABCD3-1(c/i) Risk score and the probability of ABCD3-1(c/i) itself as shown in table.1, we insert in each node its probability as mentioned. 


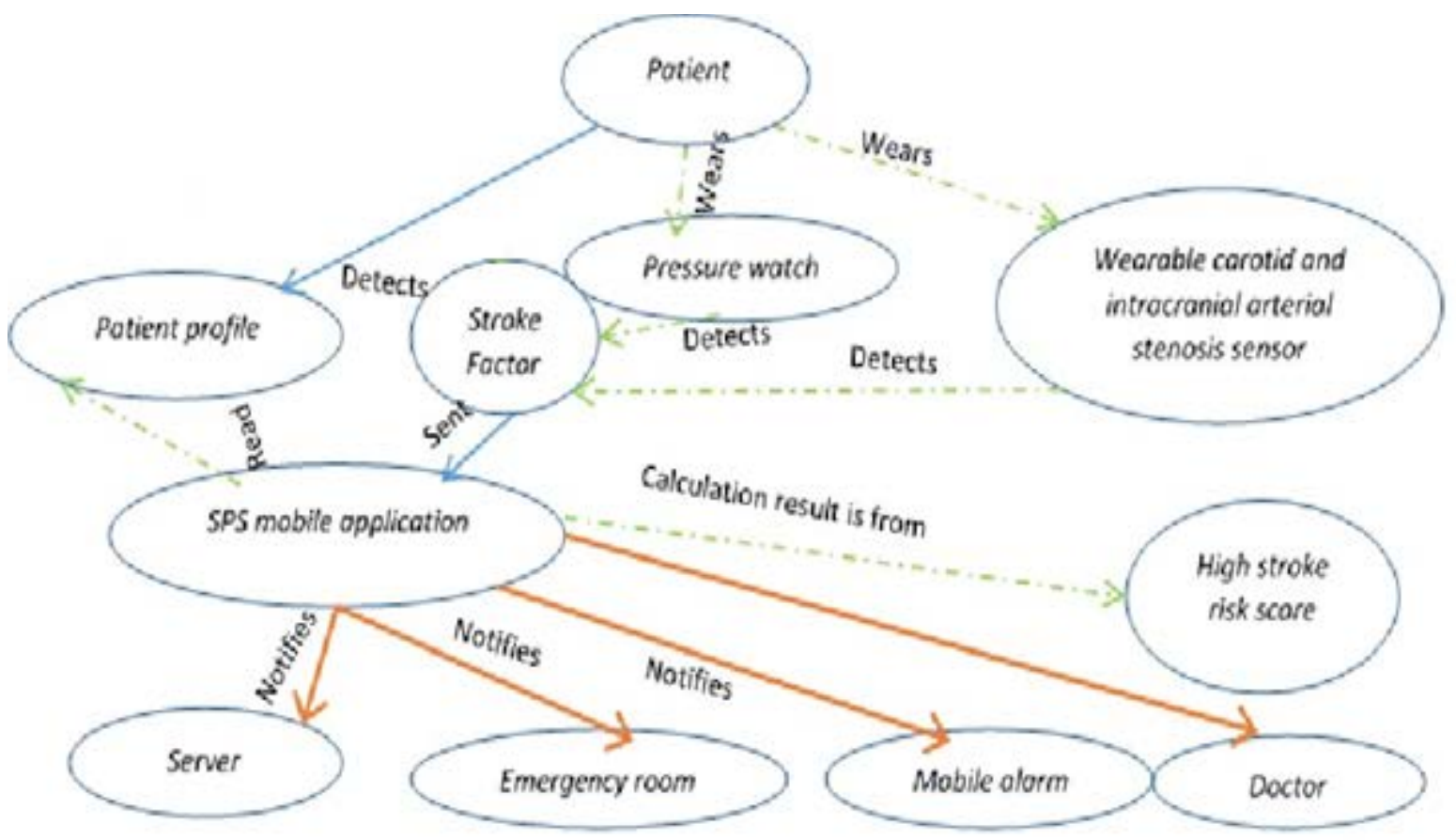

Figure 2. Bayesian belief network

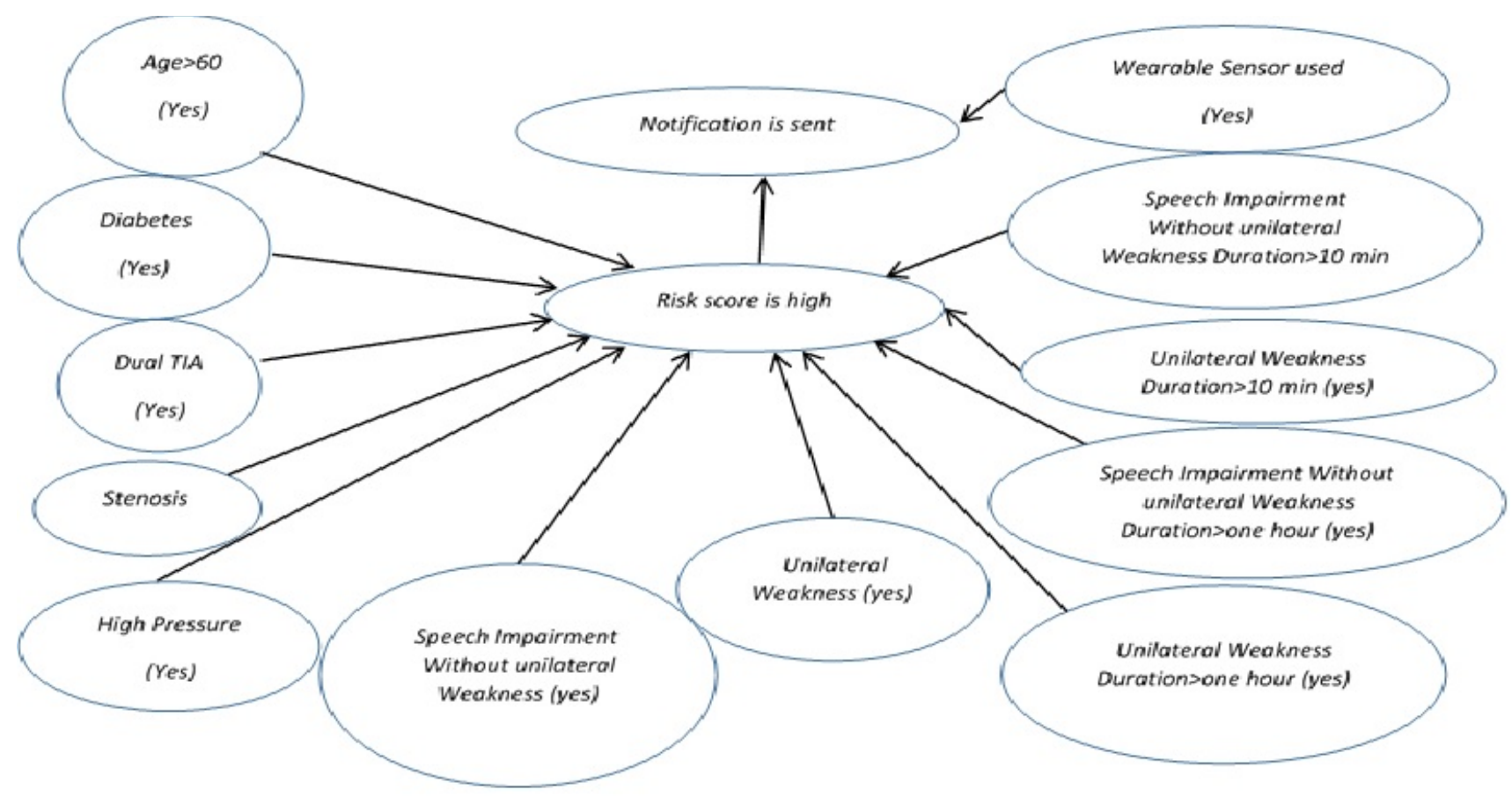

Figure 3. Updated context ontology after inference

We took the role of experts and use access visual basic code to generate an excel sheet of 513 record. These 513 records take into consideration all possible cases of parent combinations in terms of maladies and inheritance, and calculate the probability of High risk score and Mobile_App_notification using the risk score formula. Then we fill these results in the table of HighRiskscore node. The probability generated for HighRiskScore is 
$32.8 \%$ where the one in table of HighRiskscore node is $25.9 \%$, which is a reliable result.

Let's practice the adaptability of Bayesian net in Netica by changing the probability of high risk to true, we must notice the changes in the parent nodes immediately and the child node Notify will be $99 \%$ true. A new value of risk factor will have an impact on parents' nodes.

Other experience shows how the different coefficients in ABCD3-(c/i) formula which HighRiskScore is based affect the probability of highRiskScore same as if we change the probability of the parents. We tried to set "AgeGreaterThen60", "HighPressure” to true and notice the change in the probability of HighRiskScore.
Then we set "DualTIA" and "Stenosis" to true and notice how they affect HighRiskScore probability.We can easily deduce clearly importance of the formula in affecting RiskScore because the "DualTIA" and "stenosis" (higher coefficient) affect more the risk score then "AgeGreaterThen60", "HighPressure”, Netica even they have lower probabilities.

SPS application is a mobile application in reasoning layer. It's a decision based application that uses The ABCD3-1 Formula to decide if the risk of a stroke is high. The flow of the application is shown clearly in flowchart (figure 5).

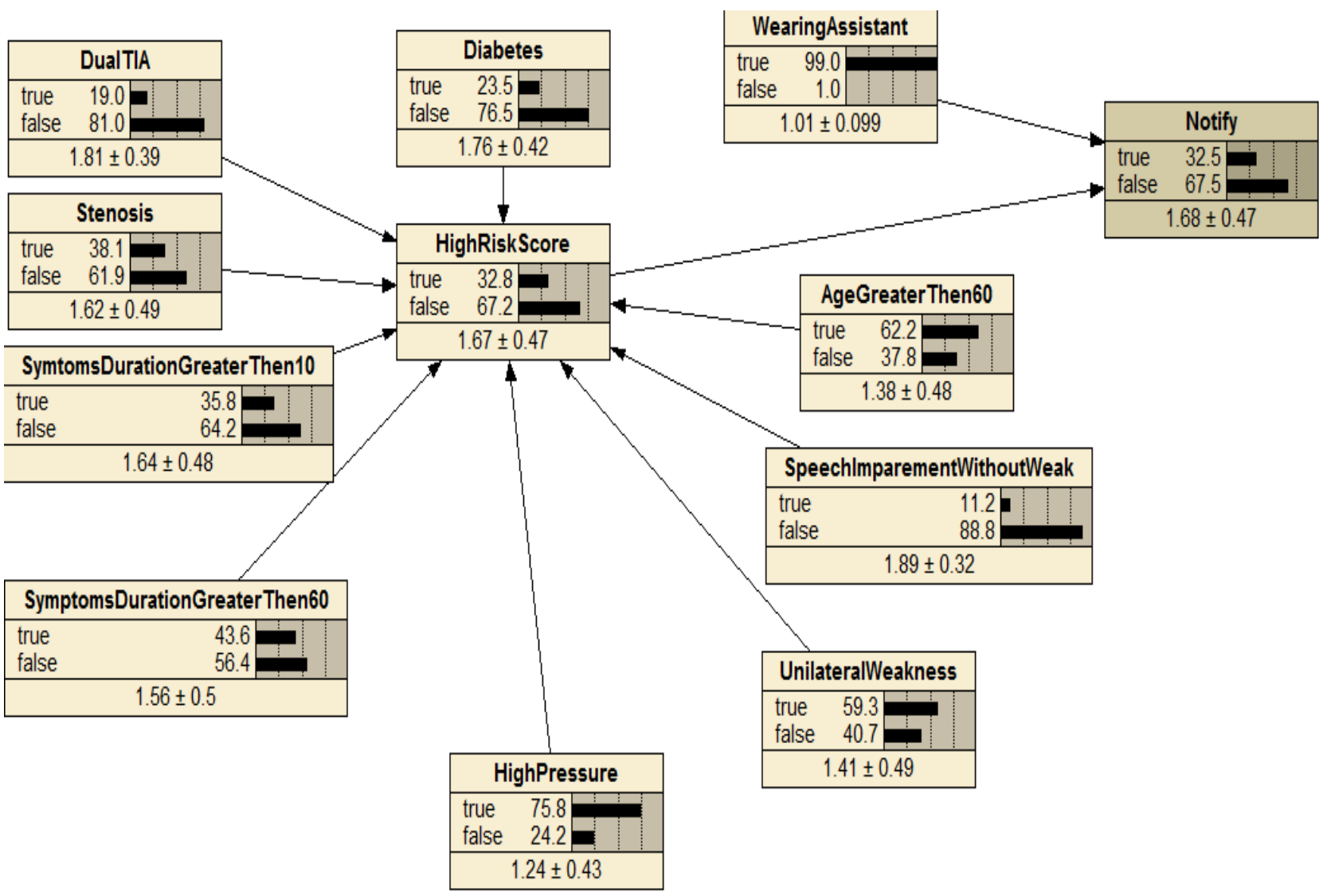

Figure 4. Bayesian network in Netica

\section{CONCLUSION}

This paper designs stroke prediction system (SPS) that monitors TIA patient to predict risk level stroke. This prediction framework is composed of many components, such as wearable assistants, mobile application, and medical team. The process and flowchart of this SPS are illustrated in figure 5: monitoring wearable assistants that aim to read patient pressure and arterial stenosis, informing mobile application interface to get patient data from many resources, analyzing them, then sending notifications in case of high risk of stroke. Patients' profiles are stored in a server side.

The core idea behind SPS is to protect patients that may be affected by a stroke due to their TIA history, age and other risk factors. SPS interface allows patient to interact with SPS application.

SPS application is a decision making application, once it predicts that feeling some symptoms may allow risk score to exceed high risk range threshold, it asks patient to check on the mobile interface the symptoms in case they occur, in addition to their duration. SPS is represented through ontology that allows us to see clearly its components and 
their influence on each other. Similar to our PHEN system where we developed Parkinson production system (PHEN), SPS uses similar model but different ontology. Since our system has certain range of uncertainty, we use its ontology to structure a Bayesian belief network. The decisions of risk score of a stroke during seven days after TIA is made by applying ABCD3-1(c/i) formula on risk factors, and compare the result to high level threshold. Moreover, we applied our Bayesian model to Netica tool where we can prove its efficiency on predicting missing probabilities and calculate the probability of high risk level for some cases. SPS is applied during seven days or more, to predict strokes and watch its effect on reducing stroke attacks. Its communication mechanism is efficient by handling patient context remotely and verifying its changes locally

Our Future work is to implement the whole stroke prediction system, especially sensors layer on all patients that arrive to the emergency room and are diagnosed as TIA patient.

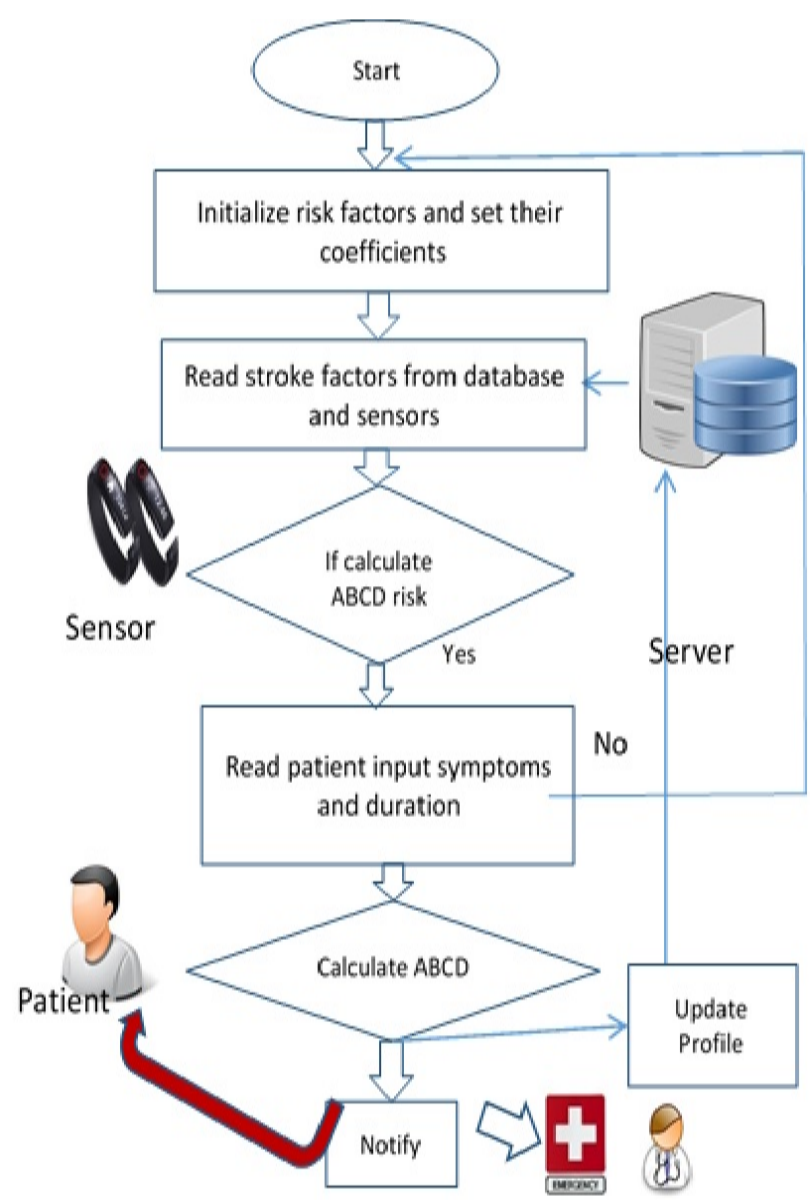

Figure 5. SPS Flowchart

\section{REFERENCES}

[1] H. Mcheick, M. Khreiss, H. Sweidan, and I. Zaarour, ”PHEN: Parkinson Helper Emergency Notification System using Bayesian Belief Network”, Springer International Publishing Switzerland, MCETEH'2015, Lecture Notes in Business Information Processing, pp. 212-223, May 12-15, 2015, Montreal, Canada.

[2] Biller J, Love BB, Schneck MJ. Vascular diseases of the nervous system: ischemic cerebrovascular disease. In: Daroff RB, Fenichel GM, Jankovic J, Mazziotta JC, eds. Bradley's Neurology in Clinical Practice . 6th ed. Philadelphia, PA: Elsevier Saunders; 2012:chap 51A.

[3] O. Neem, and A. Hein, "Clinical Decision Support with Guidelines and Bayesian Networks”, Decision Support Systems, Advances in, Book, editor Ger Devlin, chap 9, pp.117-137, March 2010, INTECH, Croatia.

[4] Germanno Teles, Carina Oliveira, Reinaldo Braga, Luiz Andrade, Ronaldo Ramos, Paulo Cunha , and Mauro Oliveira, "Using Bayesian Networks to improve the Decision-Making Process in Public Health Systems”, 2014 IEEE 16th International Conference on e-Health Networking, Applications and Services (Healthcom), pp.476-481, 2014.

[5] F. Purroy, J. Caballero, A. Gorospe, T.MJ, A. SabinJ, E. Santamarina, et al. , "Prediction of early stroke recurrence in transient Ischemic attack patients from the PROMAPA study: a comparison of prognostic risk scores”, Stroke Project of the Spanish Cerebrovascular Diseases Study Group, Cerebrovasc Dis.; 33(2):182-9, Epub Jan, 2012.

[6] P. Lavallée, E. Meseguer, H. Abboud, L.Cabrejo, J-M Olivot et al., "A transient ischemic attack clinic with round-the-clock access (SOS-TIA).feasibility and effects.”, Lancet Neurol 6: 953-960, PubMed, 2007.

[7] T. Kiyohara, M. Kamouchi, Y. Kumai, T. Ninomiya, J. Hata, S. Yoshimura, T. Ago, Y. Okada, T. Kitazono,“ABCD3 and ABCD3I Scores Are Superior to ABCD2 Score in the Prediction of Shortand Long-Term Risks of Stroke After Transient Ischemic Attack”, Epub, 2014 Feb; 45(2):418-25, 2014.

[8] H. Erdur, J. Scheitz, M. Ebinger, A . Rocco, U. Grittner, A. Meisel, P. Rothwell, M. Endres, C. Nolte "In-hospital stroke recurrence and stroke after transient ischemic attack: frequency and risk factors”. American Heart Association, 2015.

[9] G.MF, R.PM, 2007, "Risk of stroke early after transient ischemic attack: a systematic review and meta-analysis.”, Lancet Neurol 6: 1063-1072 [PubMed].

[10] Wu CM, McLaughlin K, Lorenzetti DL, Hill MD, Manns BJ, and Ghali WA., 2007, "Early risk of stroke after transient ischemic attack: a systematic review and meta-analysis.”, Arch Intern, 167(22), Med 167: 2417-2422 [PubMed].

[11] Fenton, N.E. and M. Neil, "Risk Assessment with Bayesian Networks”, CRC Press, 2012.

[12] S.O. Funtowicz and J.R. Ravetz, "Uncertainty and Quality in Science for Policy". Kluwer Academic Publishers, Dordrecht, 1990.

[13] B. Ahmed, Y.K. Lee, S. Lee and Y. Zhung, "Scenario Based Fault Detection in Context-Aware Ubiquitous Systems using Bayesian Networks”. In Proceedings of Computational Intelligence for Modelling, Control and Automation, 2005 and International Conference on Intelligent Agents, Web Technologies and Internet Commerce, International Conference on, Vol(1), pages 414, Vienna, 28-30 Nov. 2005.

[14] Netica APIs (Application Programmer Interfaces), Norsys Software Corp. 